\title{
$\leqslant$ Research Square \\ Implementing specialized vestibular physiotherapy in an emergency department: A process evaluation
}

\author{
Kelvin Ip \\ Western Health \\ Melanie Lloyd \\ Western Health \\ Allison Luscombe \\ Western Health
}

Danielle Hitch ( $\sim$ Danielle.Hitch@wh.org.au )

Deakin University - Geelong Waterfront Campus https://orcid.org/0000-0003-2798-2246

\section{Research}

Keywords: Physiotherapy, vestibular rehabilitation, Benign Peripheral Positional Vertigo, dizziness, emergency department, allied health, implementation

Posted Date: January 8th, 2021

DOI: https://doi.org/10.21203/rs.3.rs-141139/v1

License: (c) (i) This work is licensed under a Creative Commons Attribution 4.0 International License. Read Full License 


\section{Abstract}

\section{Background:}

Dizziness and vertigo-like symptoms, often caused by common peripheral vestibular disorders such as Benign Paroxysmal Positional Vertigo (BPPV), may have a significant detrimental impact on function and quality of life. The impact of these symptoms often result in Emergency Department (ED) presentations. Evidence based clinical practice guidelines strongly recommend the use of physical assessment and treatment maneuvers for the assessment, diagnosis and treatment of these symptoms. The aim of this study was to evaluate the process of implementing specialized vestibular physiotherapy in an emergency department, from the clinician perspective.

\section{Methods:}

This implementation study utilized a retrospective mixed methods process evaluation to understand how specialized vestibular physiotherapy operated in an Australian emergency department. The PARiHS Framework was embedded within the methodology and analytical approach of the study, to ensure a comprehensive approach which was closely aligned to implementation science. Nine clinicians retrospectively completed the Organizational Readiness for Change Assessment (ORCA), Acceptability of Intervention Measure (AIM), Intervention Appropriateness Measure (IAM) and Feasibility of Intervention Measure (FIM). Seven clinicians also participate in a focus group or interview.

Results: A range of barriers and facilitators to the implementation process were identified by participants, some of which spanned multiple domains of the PARiHS framework. Relationships with service leaders, champions and medical staff were found to be a key facilitator to implementation, along with a generally held perception that specialized vestibular physiotherapy was acceptable and feasible. The main barrier identified was a lack of capacity to deliver and support this innovation, both within the physiotherapy workforce and the broader multidisciplinary team.

Conclusions: This study demonstrates the process of implementation of a specialized vestibular physiotherapy team in an ED setting was generally well received by clinicians, but also involved some challenges and barriers. Services looking to implement specialized vestibular physiotherapy in the ED may refer to the recommendations arising from the findings of this study to guide their approach to innovation.

\section{Contribution To The Literature}

- Emerging evidence supports the use of specialized physiotherapy for peripheral vestibular disorders, however implementation determinants, context and processes (particularly in emergency department settings) were unknown.

- In reference to the Promoting Action on Research Implementation in Health Services (PARiHS) framework, the most influential factors for clinicians related to facilitation and recipient characteristics.

- Barriers posed by varying terminology for symptoms and interventions between patients and multidisciplinary clinicians emerged as an important priority for action.

- This study describes the experience of implementing specialized vestibular physiotherapy from the perspective of multiple clinicians, which may guide the wider dissemination of this intervention.

\section{Background}


Acute dizziness and vertigo-like symptoms are common presentations in the Emergency Department (ED). One of the common causes for such presentations is Benign Paroxysmal Peripheral Vertigo (BPPV), which is defined as calcium crystals within the inner ear being dislodged into the semi-circular canals, and thereby disrupting the vestibular system (1). Patients with BPPV commonly report subjective imbalance, dizziness or falls $(2,3)$. Clinical practice guidelines support and recommend the routine use of physical maneuvers in the clinical setting for assessment and diagnosis of $\operatorname{BPPV}(4,5)$. Failure to accurately diagnosis and treat BPPV during an initial presentation can result in negative outcomes for patients, including inappropriate use of vestibular suppressants and other medications, increased risk of falls, ongoing disruption of daily activities and decreased quality of life (68).

\section{Specialised Vestibular Physiotherapy in Emergency Departments}

Physiotherapists have skills and expertise in the management of vestibular disorders. For example, Specialised Vestibular Physiotherapy (SVP) utilizes maneuvers including the Dix-Hallpike test (DHT) and Supine Roll Test (SRT) to assess and diagnose BPPV (9). Physiotherapists are also able to administer interventions such as the CanalithRepositioning Technique (CRT) to treat BPPV when a diagnosis is confirmed. Treatment with a CRT results in symptom resolution in $67-89 \%$ of cases, in comparison to $0-48 \%$ spontaneous resolution (10).

Despite the recommendations of clinical practice guidelines, practice audit in a metropolitan Australian health service demonstrated poor uptake of this guideline (11). As a result, people presenting to the ED with BPPV may not benefit from evidence-based practice.

\section{Understanding the Implementation of Specialized Vestibular Physiotherapy}

In addition, evidence around the role of specialized vestibular physiotherapy is relatively sparse (particularly in regards to the ED), and is limited to the Australian health system. A randomized controlled trial $(n=32)$ of a physiotherapist-led home vestibular rehabilitation program (12) found the treatment group benefitted significantly from participation and continued to enjoy these improvement six months later. A more recent prospective observational study (13), found patients attending a physiotherapist-led vestibular rehabilitation service within emergency and acute services experienced significantly reduced dizziness and vertigo and significantly improved mobility (which was sustained for three months post discharge). A validated vestibular screening tool has also been developed for physiotherapists working emergency and acute settings, which was found to have good levels of sensitivity and reliability (14).

\section{Aims and Objectives}

Evidence around the role of physiotherapists in the ED to provide evidence-based management of BPPV is therefore in an early stage of development (15). While preliminary evidence regarding the outcomes of this intervention are encouraging, there remains a lack of knowledge around the implementation of these novel services in the ED setting. A greater understanding of how to successfully introduce and sustain specialized vestibular physiotherapy will be crucial to the scaling up and ongoing evaluation of its impact on with people experiencing BPPV.

\section{Aim and Objectives}

The aim of this study was to evaluate the process of implementing specialized vestibular physiotherapy in an ED, from the clinician perspective. The implementation objective was to understand barriers and facilitators associated with the implementation process, and develop recommendations for other services looking to introduce specialized 
vestibular physiotherapy in the ED setting. This evaluation was undertaken alongside a pilot study of its safety and feasibility, which has been reported elsewhere (16).

\section{Materials And Methods}

\section{Design}

Ethical approval to proceed with this study was granted by the local health service ethics board (HREC/18/WH/120). This study utilized a retrospective mixed method process evaluation to address the study aim. Process evaluations aim to build workforce capacity in the use of evidence informed interventions, and explore not only 'what works' but 'why' (17). This approach frequently employs mixed methods, and while it can be time and resource intensive, there is increasing recognition of the significant role of process evaluation in understanding the reality of using complex interventions in practice (18). In particular, process evaluations can provide valuable information about the lived experience of implementation, including the barriers to and facilitators of effective translation of evidence into daily practice (19).

To ensure the study drew on established implementation theory, the Promoting Action on Research Implementation in Health Services (PARiHS) framework was used as an organizing theoretical structure. The PARiHS framework is designed to guide the implementation of evidence-based practice and is widely used in implementation across a range of disciplines (20). The PARiHS consists of four domains - the characteristics of the setting or context (Context), the way in which evidence is facilitated (Faciliation), the individuals and team engaged in implementation (Recipients) and the quality and type of evidence (Innovation) - all of which are believed to influence the success of implementation. In this framework, successful implementation is proposed to result from the interaction between facilitation, innovation, recipient characteristics and context (21).

\section{Context}

Specialized vestibular physiotherapy was implemented in the ED of a tertiary metropolitan Australian health service. The service operates three acute public hospitals, and this study was undertaken at a single hospital site. This hospital provides a range of support, including acute and community-based services to aged, adult, paediatric and maternity populations. The local community has a population of approximately 800,000 people, is culturally and linguistically diverse, and is located in one of the fastest growing regions in Australia (22). Approximately 250 patients present to the hospital's ED daily (11).

\section{Implementation Strategy}

A specialized vestibular physiotherapy service was implemented in the ED over the second half (8 weeks) of the 16week recruitment period for the feasibility study. It was made available to eligible patients during clinician business hours (8am to 4 pm), Monday to Friday due to available resources. Each weekday morning, a study investigator attended the ED and reviewed the triage record of admissions since 4 pm the preceding day to identify patients with specific symptoms (dizziness, vertigo, imbalance, loss of balance, or fall). If any identified patients remained in the $E D$, the investigator consulted with the medical officer to determine whether the patient was suitable for assessment and intervention by this service. The investigator also periodically reviewed the triage notes throughout the day to identify further qualifying patients who presented during business hours.

\section{Description of the Intervention}


The specialized vestibular physiotherapy service targeted adults aged $\geq 18$ presenting to the ED with symptoms of dizziness, vertigo or imbalance. Exclusion criteria included; 1) Primary diagnosis by medical officer at initial assessment (or thereafter) with a clear non-vestibular cause; 2) Patients unable to understand instructions and/or willingly participate in vestibular assessment with physiotherapist (due to language, cognition, symptoms or any other reason); and 3) Patients presenting to ED outside of specialized vestibular physiotherapy service hours (16).

The SVP intervention involved assessment and treatment as per clinical practice guideline recommendations $(4,5)$ Once patient symptoms improved enough to allow safe mobilization, they were discharged from the ED with education about their condition and a referral for specialist physiotherapy follow-up as an outpatient. A range of outcomes from the specialized vestibular physiotherapy service were evaluated as part of the feasibility study (16), and these are provided in Additional file 1.

\section{Eligibility and recruitment for Implementation Study}

Eligible participants for this study included physiotherapy, medical and nursing clinicians from the ED, and inpatient acute care and community rehabilitation clinicians who were impacted by the trial implementation of the specialized vestibular physiotherapy service, and therefore considered stakeholders. Clinicians were identified as key stakeholders and invited to participate if they were directly involved in the clinical management of patients diagnosed with peripheral vestibular dysfunction (either in the acute or sub-acute setting).

All eligible clinicians were invited to participate by email, and asked to return a written consent form. Participants were able to indicate on the consent form what modes of data collection they wished to participate in (i.e. ORCA only, interview/focus group only, ORCA and interview/focus group). As per the feasibility study to which this evaluation was linked, the sample size was informed by purposive selection, which identified participants with specific experience of the implementation process under investigation. The study sample included 11 staff members (4 care coordinators, 3 doctors, 5 physiotherapists, and 1 nurse), as not everyone invited chose to participate.

\section{Outcomes of Implementation}

The outcomes of interest in this process evaluation were organizational readiness for change, and clinician perceptions of the implementation process (including aspects such as acceptability, appropriateness, feasibility and contextual factors). Data related to patient perceptions of the implementation process were also collected, and will be reported elsewhere. Four outcomes measures were used to gather quantitative data- the Organizational Readiness for Change Assessment (ORCA) (23), Acceptability of Intervention Measure (AIM), Intervention Appropriateness Measure (IAM) and Feasibility of Intervention Measure (FIM) (24).

The ORCA is a 77-item checklist designed to operationalize the constructs of the PARiHS framework (23). The psychometric properties of the ORCA have been established, with its reliability and validity both found to be acceptable across most sub-scales (apart from those related to evidence). In this study, the ORCA was used retrospectively to enable clinicians to identify needs and conditions they perceived to be important to the process of specialized vestibular physiotherapy implementation.

The AIM/IAM/FIM is a combined suite of implementation outcome measures, developed to monitor and evaluate the success of implementation initiatives. The measurement properties of these brief scales have received preliminary confirmation, with reasonable structural validity, known groups validity, test-retest reliability and sensitivity to change indicated (24). Higher scores are interpreted as indicating greater acceptability, appropriateness and feasibility, and scale scores are calculated with mean responses. 
Qualitative data was collected via semi-structured interviews and focus groups. A bespoke set of interview prompts were developed, as no suitable existing prompts or schedules were available. The schedules were developed from previously published reflective questions for facilitators using the PARiHS framework (20), and began with prompts which specifically focused on each assessment and treatment component in the clinical practice guidelines. The schedule then become more general in approach, enabling clinicians to reflect and comment on the implementation process as a whole. All focus groups and interviews were digitally recorded, and transcribed verbatim for analysis. The interview prompts have been provided as Additional file 2.

\section{Data Analysis}

Data was analyzed using a convergent parallel model of mixed methods. This model entails the concurrent collection of quantitative and qualitative data, and values both forms equally when they are combined to produce the overall interpretation (25). All data was transformed to align with the PARiHS Framework, to maintain alignment with implementation theory and also enable more direct contrast and comparison.

Ordinal quantitative responses from the outcome measures were aligned to the domains of the PARiHS Framework. The process of alignment was undertaken by reviewing definitions and descriptions of the four domains (Context, Facilitation, Recipients and Innovation), and assigning each item on the ORCA and AIM/IAM/FIM to its related domain. This process was initially undertaken by one researcher (DH) before being independently reviewed and confirmed by a second researcher $(\mathrm{KI})$. The alignment between the items and the domains are shown in Additional file 3, and not all aspects of the PARiHS Framework were addressed by the quantitative question. This data was analyzed using SPSS Version 25.0, and reported using descriptively to illustrate the proportion of responses.

Qualitative transcriptions were subjected to content analysis, in alignment with the PARiHS framework. A codebook was developed to ensure consistency in analysis, providing definitions for all concepts related to the PARiHS Framework and examples of its presence in the dataset. The codebook has been provided as Additional file 4. Data were systematically coded and categorized to identify trends and patterns, their frequency and relationships. The analysis was completed independently by two researchers $(\mathrm{KI}, \mathrm{DH})$, and compared for consistency. A third researcher ( $\mathrm{ML}$ or $\mathrm{AL}$ ) also reviewed all coding, and the few instances of disagreement identified were resolved by consensus. The reporting of this study meets the requirements of the Standards for Reporting Implementation Studies (StaRI) statement (26), which has been included as Additional file 5.

\section{Results}

Nine participants responded to the quantitative outcome measures, and seven clinicians participate in one focus group and one individual interview. The integrated findings from both forms of data are presented in alignment with the PARHiS Framework.

\section{Facilitation}

As shown below in Fig. 1, clinicians agreed or strongly agreed that senior leadership practices and champions had a positive impact on the process of implementing specialized vestibular physiotherapy in the ED. However, there was more uncertainty around perceptions of general leadership roles, implementation progress, implementation communication and evaluation plans.

This uncertainty may have been reflected in the fact that relatively few references to facilitation were identified in the qualitative data. Participants provided their perceptions around the qualities of facilitation that contributed to the 
successful implementation of the specialized vestibular physiotherapy service, especially highlighting a need for exceptional communication and inter-personal skills. In their view "it really came down to [facilitator] ability to communicate and develop rapport with the multidisciplinary team down in ED". In addition to possessing the skills and knowledge in providing assessment, diagnosis, and treatment for BPPV, these interpersonal skills were identified as enabling healthy relationships with staff and patients. Participants described needing a facilitator "who's confident and can communicate confidently with medical teams and patients".

\section{Innovation}

The majority of participants $(n=6,66.7 \%$ ) perceived the strength of the evidence for specialized vestibular physiotherapy for BPPV as very strong. However, there was less confidence expressed in the strength of evidence from the perspective of clinical experts, with $37.5 \%(n=3)$ identifying neutral or weak perceptions. The majority of participants $(n=6,82.7 \%)$ perceived specialized vestibular physiotherapy as fitting the services priorities and needs very or extremely well, and all participants believed this service successfully addresses and treats BPPV. As shown below in Fig. 2, there were few neutral or disagree responses to items on the ORCA related to the innovation. There was a significant amount of qualitative data in relation to Innovation, which consistently emphasized the benefits and advantages of this service to patients and the service. The specialized vestibular physiotherapy service was perceived by many participants as having the positive impact on access and flow in the ED; "there was someone there who could actually do the treatment and that meant free them up maybe to do something else because you know ED is such a crazy place". While there were differences in opinion about the scope of this innovation, it was also believed to offer an opportunity to a wider range of patients in a cost effective manner; "The doctors felt quite strongly that we should really be focusing on those people that came with a classic vertigo presentation ... you can get people that might just come in with a fall and no background of dizziness or vertigo, but that's what's causing it ... if you've had a fall, the morbidity and mortality rates and those sorts of things, it's having quality of life as well".

Participants also reported significant levels of satisfaction with the implementation of this innovation, which they did not perceive as burdensome or overly difficult. A key aspect of these perceptions was the acknowledgement that; "People were getting the right care in ED when they needed to ... I really enjoyed being able to treat patients using evidence based practice, doing things that we know work and actually make a difference". Participants also appreciated the opportunity to be proactive rather than reactive in responding to these patients needs, and recognized the importance of remaining abreast of, and responsive to, emerging evidence in this area; "New recommendations against that came out in the most recent iteration of the guidelines for not using upright positioning because previously we used to tell people to sit up for six to eight hours after they've been treated and we don't do that anymore".

Terminology emerged as an important issue to consider during implementation. Elements of these physiotherapy interventions have multiple names, which were at times confusing for the multidisciplinary team. The interventions also needed additional explanation to both colleagues and patients, because "it looks a bit awkward. It looks strange, and there's a lot of education that goes around that". However, a more consistently encountered challenge was communicating about BPPV with the culturally diverse patients attending this service; "the terms delirium, dizziness, light-headedness or vertigo can be used interchangeable, depending on your cultural background, your belief systems, your health literacy. And then you add that layer as many do have English as their second language, they all need the complexity of an interpreter to then try and sift through those". Another consideration when implementing this innovation was the sporadic nature of BPPV presentations to the ED, which posed some challenges to resourcing the specialized vestibular physiotherapy service; "We had weeks where we'd have eight or 
nine patients, and then weeks where we'd have none, so juggling not being able to predict was an added stress". A solution to this problem implemented in this service was to have these physiotherapists available as a float for other wards and service at times when they were not required in the ED.

\section{Recipients}

While the recipients of the innovation were the patients who presented to ED with BPPV, the recipients of this implementation process were clinicians within the service. The majority of participants strongly believed the specialized vestibular physiotherapy service offered to patients with BPPV was appropriate, acceptable and feasible in the ED. As shown in Fig. 3, there were no participants disagreed and very few held a neutral view.

The Recipients domain was most frequently cited in the qualitative data of this study, and three sub-themes were identified. Firstly, local staff members within the ED were receptive with the implementation of the service; "I did notice, happy smiles which you don't get down here very often". These factors relied on the formation of relationship with multidisciplinary colleagues, which was a particular focus given the novelty of the specialized vestibular physiotherapy service; "Having to re-establish identity as who you are and what your skills are and to work out who people are to be able to have that relationship to go from ... having to meet and greet with new people". The second identified sub-theme was the medical team in ED played a crucial role in the success of implementation. Enthusiasm and investment from medical staff was a significant facilitator for both referrals and integration with other ED activities; "The ED doctors seem to be buying in ... we certainly needed buy in from the key physicians, so neurology, general medicine, had a multidisciplinary input".

Despite the evidence supporting the role of physiotherapy in managing BPPV, many physiotherapists identified a general lack of knowledge and skill within the profession as a barrier to treating this patient population. While formalized continuing professional development is available, informal training remains a common path to competence in this area of practice "I've never done a specialized course, I really learned from seeing clinicians, or from doing doubles and stuff". The need to develop knowledge and skills in this area was not unique to physiotherapy, with participants also observing variable practice amongst other colleagues; "I have seen the most weird, I wouldn't say wonderful, variations of hallpikes ... what's everyone's understanding of what is actually a hallpike? And therefore your interpretation of results and what you do with it is not all on the same page as well". Due to the emerging nature of specialized vestibular physiotherapy services in the ED, participants also perceived a more general lack of awareness around what they may offer; "Doctors probably don't know what we offer and that's something that we're trying to fix".

\section{Context (Local, Organisational and External Health System)}

As shown below in Fig. 4, most participants perceived the local ED team and broader organization were committed to providing patients with effective treatment and were receptive to the implementation process. Their perceptions of context also confirmed the crucial role that clinical leaders play in implementation, by establishing goals and providing feedback to staff. However, perceptions of the organization's ability to provide adequate resources to sustain the specialized vestibular physiotherapy service in the longer term were more mixed.

Qualitative data related to this domain identified some practical measures required to support the sustained the specialized vestibular physiotherapy service, including greater clarity around referrals and pathways; "For a goal based service you need to have this information if you're going to refer to us". Formal policies or regulations to formalise the role of physiotherapists in this setting are yet to be developed, and were recognized as an important resource for sustained implementation: "I think as an organization we need to look more broadly at how it would fit in, so it's not just this little service that operates in ED, that we set the pathways". The key role of medical staff in this implementation process was reaffirmed, as was the value of providing some specific education around the form and 
potential outcomes of specialized vestibular physiotherapy; "I think it was really important that we did some education sessions with the doctors".

Adequate resourcing was another important influence on sustainability commonly discussed by the participants. To provide the specialized vestibular physiotherapy service in the ED within existing and finite resources, some participants proposed a broader reconfiguration would be required in the broader physiotherapy department; "where we can, withdraw our services from elsewhere when they're not necessarily being used that effectively, or what extra resources we would need to be able to run that kind of program". The need for the service to be offered seven days a week was also recognized, to ensure all patients presenting with BPPV had the opportunity to access these assessments and interventions; "It needs to be a seven day service ... because obviously dizziness happens seven days a week'.

The participants also recognized the potential impact of the specialized vestibular physiotherapy service on the broader continuum of care, particularly after the patients have been discharged from the ED. While patients were referred for continuing physiotherapy, the role of general practitioners emerged as a strong theme in the data. While some were supportive of their patients receiving physiotherapy for their BPPV, others did not perceive this to be an appropriate intervention; "We talked about how many people were seen then went back to the GP and the GP said "that's a load of rubbish. It's complete rubbish, don't bother doing that. It's wrong". Widening the focus of the service to form partnerships with community providers was identified as an important priority for the future, to enable it benefits to be fully realized.

\section{Discussion}

The findings of this study have provided a clinician's perspective on the process of implementing specialized vestibular physiotherapy in an emergency department. The PARiHS Framework describes successful implementation as a function of facilitation (innovation + recipients + context) (21). Successful implementation is demonstrated by evidence of 1 ) achievement of the implementation goal; 2) the innovation being embedded in practice; 3 ) engaged and motivated individuals, teams and stakeholders; and 4) minimal variation across settings. While the fourth benchmark was not applicable to this study, the findings presented here are supportive of the other identified markers of success.

A variety of barriers and facilitators to the implementation process were identified by participants, some of which spanned multiple domains of the PARiHS framework. For example, the crucial role of service leaders and champions with excellent interpersonal skills in enabling the implementation of this specialized vestibular physiotherapy service was discussed in relation to both facilitation and context. Service leaders have a significant impact on the attitudes, priorities and behaviours of their colleagues (27), and this finding consolidates existing evidence in this area. The use of champions has also been found to enable successful implementation in healthcare (28), however similar to previous innovations the position was not operationalized formally in this study. Along with the development of policies and procedures, the development of recruitment and workforce resources to support this service will be important to its ongoing sustainability.

Medical staff were particularly identified as key contacts both within and beyond the ED department, which reflects their core role within the Australian health system. Their influence on the implementation process could be positive or negative, depending on their perception of the value of, and evidence base for specialized vestibular physiotherapy. The influence of workplace cultures on hierarchical relationships in acute health are well recognized, but may be a barrier to implementation when they result in the formation of silos or dysfunctional care pathways 
(29). Medical staff (and nursing staff) were gatekeepers for this service, as they triaged and determined whether appropriate patients were referred for assessment and treatment. This is a common feature of the allied health practice context, and demands multidisciplinary collaboration which adds to the complexity of implementation of every innovation (30). The findings of this study indicated these relationships were successfully negotiated during this implementation process.

The ED team and other stakeholders were also reported to be accepting and supportive of the new service, which was attributed to a shared recognition of the potential for positive outcomes for patients and the ED itself. The quantitative and qualitative data was also consistent in its support for the perceived acceptability, appropriateness and feasibility of the specialized vestibular physiotherapy service in the ED. Developing a shared vision or shared goals for change is an effective strategy for promoting buy-in and participation whenever practice transformation is attempted (31). However, in this study many participants reported feeling unsure about the implementation plan and evaluation plan for specialized vestibular physiotherapy in the ED. Support from the multidisciplinary team might therefore be consolidated or developed even further through more explicit reporting of goals and evaluation results in the future.

A key barrier identified in the study was varying levels of knowledge around the role of physiotherapy with these patients, and the effectiveness of the interventions themselves. This lack of awareness and different opinions around the scope of practice may lead to missed opportunities for referrals, and less than optimal execution of the specialized vestibular physiotherapy service. Some physiotherapists also described a lack of confidence around providing these assessments and interventions, as they have not received formalized training. A lack of capacity within the physiotherapy profession may also therefore limit the potential scaling up of these services, as there won't be sufficient staff available to deliver the service. A previous evidence synthesis has found that educational strategies may be effective in promoting implementation, but only if they directly address barriers and facilitators relevant to the innovation (32). The findings of this study may therefore provide a basis for the design of tailored education and training for all stakeholders relevant to the implementation of these services.

In summary, the findings of this study indicate several key recommendations for the future implementation of specialized vestibular physiotherapy in the ED. Investment in the formation and maintenance of relationships with all stakeholders will enhance the chances of success, particularly in regards to senior leadership, champions and medical colleagues. A shared vision of the goals and outcomes of these services for both patients and the ED service itself should be explicitly stated, and form the basis of ongoing evaluation to ensure this innovation meets the needs of its stakeholders. The process of ensuring that goals and outcomes are explicit should also include a focus on consistent terminology (for use with both patients and colleagues) and the availability of or need for resources. Efforts to increase capacity within services for specialized vestibular physiotherapy should be tailored to meet the different needs of the physiotherapy workforce and multidisciplinary team members. Finally, formal processes, procedures and policies to embed the service into existing organizational systems (such as position descriptions and referral pathways) should be developed as early in the implementation process as possible, and regularly reviewed by all stakeholders.

\section{Strengths and Limitations}

This study has several strengths, beginning with the collection of data from participants across different disciplines within the ED. This provided a wholistic perspective of the success of implementation of the service, and supported an understanding of the process from multiple points of view. A rigorous approach to the qualitative analysis was 
also undertaken, including multiple coding and peer review. The use of the PARiHS Framework in both methodological design and analysis also ensured the study retained its focus on implementation, and explored the process in a comprehensive and evidence based manner.

However, the small sample size in this study also introduces some significant limitations to the interpretation of the findings. During the focus groups and interviews, the majority of participants were physiotherapists despite an open invitation to other stakeholders, and therefore this data may not be representative of the perceptions of all stakeholders. This study is also only reflective of the implementation process at a single campus of a single health service within the Australian health service context. Finally, the collection of data retrospectively may have missed changes in perception and experience that occurred over time during the implementation process.

\section{Implications}

This study provides preliminary data around the implementation process for specialized vestibular physiotherapy in ED settings, and demonstrates this is a complex and sometimes challenging experience for clinicians and teams. As the first study to address this aspect of the topic, it extends the existing evidence base in a new direction that is important to the practical development of these services. This study also provides a basis for future research, education and practice, as a guide for clinicians and service providers as this intervention is scaled up. The recommendations presented above can assist physiotherapists and their colleagues enact the implementation of specialized vestibular physiotherapy more effectively, after adapting and modifying the innovation to meet their own local needs.

\section{Conclusion}

The findings of this study show the process of implementation of a specialized vestibular physiotherapy team in an ED setting was generally perceived positively by clinicians. Recommendations for future implementation and development were also identified including building relationships with stakeholders, developing an explicit shared vision, explicitly stating goals and intended outcomes, embedding the innovation in organizational processes, procedures and policies, and increasing workforce capacity to delivery and support specialized vestibular physiotherapy for patients with BPPV presenting to the ED. The outcomes of this study indicate this innovation has significant potential to make a meaningful impact on both the function of the ED team and the lives of patients presenting with dizziness.

\section{Abbreviations}

AIM - Acceptability of Intervention Measure

BPPV - Benign Paroxysmal Positional Vertigo

CRT - Canalith-Repositioning Technique

DHT - Dix-Hallpike Test

ED - Emergency Department

FIM - Feasibility of Intervention Measure

IAM - Intervention Appropriateness Measure

Page $11 / 17$ 
ORCA - Organizational Readiness for Change Assessment

PARiHS - Promoting Action on Research Implementation in Health Services framework

SRT - Supine Roll Test

SVP - Specialised Vestibular Physiotherapy

\section{Declarations}

\section{Ethics approval and consent to participate}

Ethical approval to proceed with this study was granted by the Western Health Ethics Committee (HREC/18/WH/120). Explicit written consent was obtained from all participants.

\section{Consent for publication}

Not applicable.

\section{Availability of data and materials}

The datasets used and/or analysed during the current study are available from the corresponding author on reasonable request

\section{Competing interests}

The authors declare that they have no competing interests.

\section{Funding}

The parent study was funded by an internal health service grant, however this implementation study was completed using existing resources.

\section{Authors' Contributions}

Conception or design of the work - KI, ML, AL, DH

Data collection - $\mathrm{KI}, \mathrm{AL}, \mathrm{DH}$

Data analysis and interpretation - KI, ML, $\mathrm{AL}, \mathrm{DH}$

Drafting the article $-\mathrm{KI}, \mathrm{DH}$

Critical revision of the article $-\mathrm{KI}, \mathrm{ML}, \mathrm{AL}, \mathrm{DH}$

Final approval of the version to be published $-\mathrm{KI}, \mathrm{ML}, \mathrm{AL}, \mathrm{DH}$

\section{Acknowledgments}

The authors thank the staff of the Sunshine Hospital Emergency and Physiotherapy Departments for their support of the Dizzy-PT study. Anne-Maree Kelly, Harin Karunajeewa, Sharon Klim, Tissa Wijeratne and Catherine Grant 
assisted with the study design and implementation of the parent comparative study.

\section{References}

1. Parnes LS, Agrawal SK, Atlas J. Diagnosis and management of benign paroxysmal positional vertigo (BPPV). Canadian Medical Association Journal. 2003;169(7):681-93.

2. von Brevern M, Radtke A, Lezius F, Feldman M, Ziese T, Lempert T, et al. Epidemiology of benign paroxysmal positional vertigo: a population based study. Journal of Neurology, Neurosurgery \& Psychiatry. 2007;78(7):7105.

3. Abbott J, Tomassen S, Lane L, Bishop K, Thomas N. Assessment for benign paroxysmal positional vertigo in medical patients admitted with falls in a district general hospital. Clinical Medicine. 2016;16(4):335.

4. Bhattacharyya N, Gubbels S, Schwartz S, Edlow J, El-Kashlan H, Fife T, et al. Clinical Practice Guideline: Benign Paroxysmal Positional Vertigo (Update) Executive Summary. Otolaryngology-Head and Neck Surgery. 2017;156(3):403-16.

5. Fife T, Iverson D, Furman J, Baloh R, Tusa R, Hain T, et al. Practice Parameter: Therapies for benign paroxysmal positional vertigo (an evidence-based review): Report of the Quality Standards Subcommittee of the American Academy of Neurology. Neurology. 2008;70(22):2067-74.

6. Wang A, Yu D, Song N, Su K, Yin S. Delayed diagnosis and treatment of benign paroxysmal positional vertigo associated with current practice. European Archives of Oto-Rhino-Laryngology. 2014;271(2):261-4.

7. Polensek S, Tusa R, Sterk C. The challenges of managing vestibular disorders: a qualitative study of clinicians' experiences associated with low referral rates for vestibular rehabilitation. International Journal of Clinical Practice. 2009;63(11):1604-12.

8. Oghalai J, Manolidis S, Barth J, Stewart M, Jenkins H. Unrecognized benign paroxysmal positional vertigo in elderly patients. Otolaryngology-Head and Neck Surgery. 2000;122(5):630-4.

9. Power L, Murray K, Szmulewicz D. Characteristics of assessment and treatment in Benign Paroxysmal Positional Vertigo (BPPV). Journal of Vestibular Research. 2020;30(1):55-62.

10. Tusa R, Herdman S. BPPV: Controlled trials, contraindications, post-manoeuvre instructions, complications, imbalance. Audiological Medicine. 2005;3(1):57-62.

11. Lloyd M, Mackintosh A, Grant C, McManus F, Kelly A-M, Karunajeewa H, et al. Evidence-based management of patients with vertigo, dizziness, and imbalance at an Australian metropolitan health service: an observational study of clinical practice. Physiotherapy Theory and Practice. 2020;36(7):818-25.

12. Enticott J, Vitkovic J, Reid B, O'Neill P, Pain M. Vestibular Rehabilitation in Individuals with Inner-Ear Dysfunction: A Pilot Study. Audiology and Neurotology. 2008;13(1):19-28.

13. Stewart V, Mendis M, Low Choy N. Clinical effectiveness of a physiotherapy-led, hospital-based vestibular service. Otorinolaringologia 2019;69(1):1-8.

14. Stewart V, Mendis M, Rowland J, Low Choy N. Construction and Validation of the Vestibular Screening Tool for Use in the Emergency Department and Acute Hospital Setting. Archives of Physical Medicine \& Rehabilitation. 2015;96(12):2153-60.

15. Stewart V. Improving Screening and Physiotherapy Management of Vestibular Disorders in the Hospital Setting. Banyo, NSW: Australian Catholic University; 2018.

16. Lloyd M, Luscombe A, Grant C, Karunajeewa H, Klim S, Wijeratne T, et al. Specialised vestibular physiotherapy in the emergency department: A pilot safety and feasibility study. Emergency Medicine Australasia. 
2020;32(5):860-3.

17. Haynes A, Brennan S, Carter S, O'Connor D, Schneider C, Turner T, et al. Protocol for the process evaluation of a complex intervention designed to increase the use of research in health policy and program organisations (the SPIRIT study). Implementation Science. 2014;27(9):113.

18. Limbani F, Goudge J, Joshi R, Maar MA, Miranda JJ, Oldenburg B, et al. Process evaluation in the field: global learnings from seven implementation research hypertension projects in low-and middle-income countries. BMC Public Health. 2019;19(1):953.

19. Schneider M, Hall WJ, Hernandez AE, Hindes K, Montez G, Pham T, et al. Rationale, design and methods for process evaluation in the HEALTHY study. Int J Obes (Lond). 2009;33 Suppl 4(Suppl 4):S60-S7.

20. Harvey G, Kitson A. PARIHS revisited: from heuristic to integrated framework for the successful implementation of knowledge into practice. Implementation Science. 2016;11:33.

21. Stetler CB, Damschroder LJ, Helfrich CD, Hagedorn HJ. A Guide for applying a revised version of the PARIHS framework for implementation. Implementation Science. 2011;6(1):99.

22. Australian Bureau of Statistics. 2016 Census Community Profiles - General Community Profile 2016. Available from:

https://quickstats.censusdata.abs.gov.au/census_services/getproduct/census/2016/communityprofile/21301? opendocument.

23. Helfrich CD, Li Y-F, Sharp ND, Sales AE. Organizational readiness to change assessment (ORCA): Development of an instrument based on the Promoting Action on Research in Health Services (PARIHS) framework. Implementation Science. 2009;4(1):38.

24. Weiner B, Lewis C, Stanick C, Powell B, Dorsey C, Clary A, et al. Psychometric assessment of three newly developed implementation outcome measures. Implementation Science. 2017;12:108.

25. Cresswell J, Plano Clark V. Designing and conducting mixed methods research. 3rd ed. New York: SAGE; 2018.

26. Pinnock H, Barwick M, Carpenter C, Eldridge S, Grandes G, Griffiths C, et al. Standards for Reporting Implementation Studies (StaRI) statement. British Medical Journal. 2017;356:i6795.

27. Hernandez M, Eberly M, Avolio B, Johnson M. The loci and mechanisms of leadership: exploring a more comprehensive view of leadership theory. Leadership Quarterly. 2011;22:1165-85.

28. Miech E, Rattray N, Flanagan M, Damschroder L, Schmid A, Damush T. Inside help: An integrative review of champions in healthcare-related implementation. SAGE Open Medicine. 2018;6(2050312118773261).

29. Braithwaite J, Clay-Williams R, Vecellio E, Marks D, Hooper T, Westbrook M, et al. The basis of clinical tribalism, hierarchy and stereotyping: a laboratory-controlled teamwork experiment. BMJ Open. 2016;6(7):e012467.

30. Hitch D, Pepin G, Lhuede K, Giles S. Development of the Translating Allied Health Knowledge (TAHK) Framework. International Journal of Health Policy and Management. 2019;8(7):412-23.

31. Doten-Snitker K, Margherio C, Litzler E, Ingram E, J Developing a Shared Vision for Change: Moving toward Inclusive Empowerment. Research in Higher Education. 2020:https://doi.org/10.1007/s11162-020-09594-9.

32. Powell B, Fernandez M, Williams N, Aarons G, Beidas R, Lewis C, et al. Enhancing the Impact of Implementation Strategies in Healthcare: A Research Agenda. Frontiers in public Health 2019;7(3):doi:

10.3389/fpubh.2019.00003.

\section{Figures}




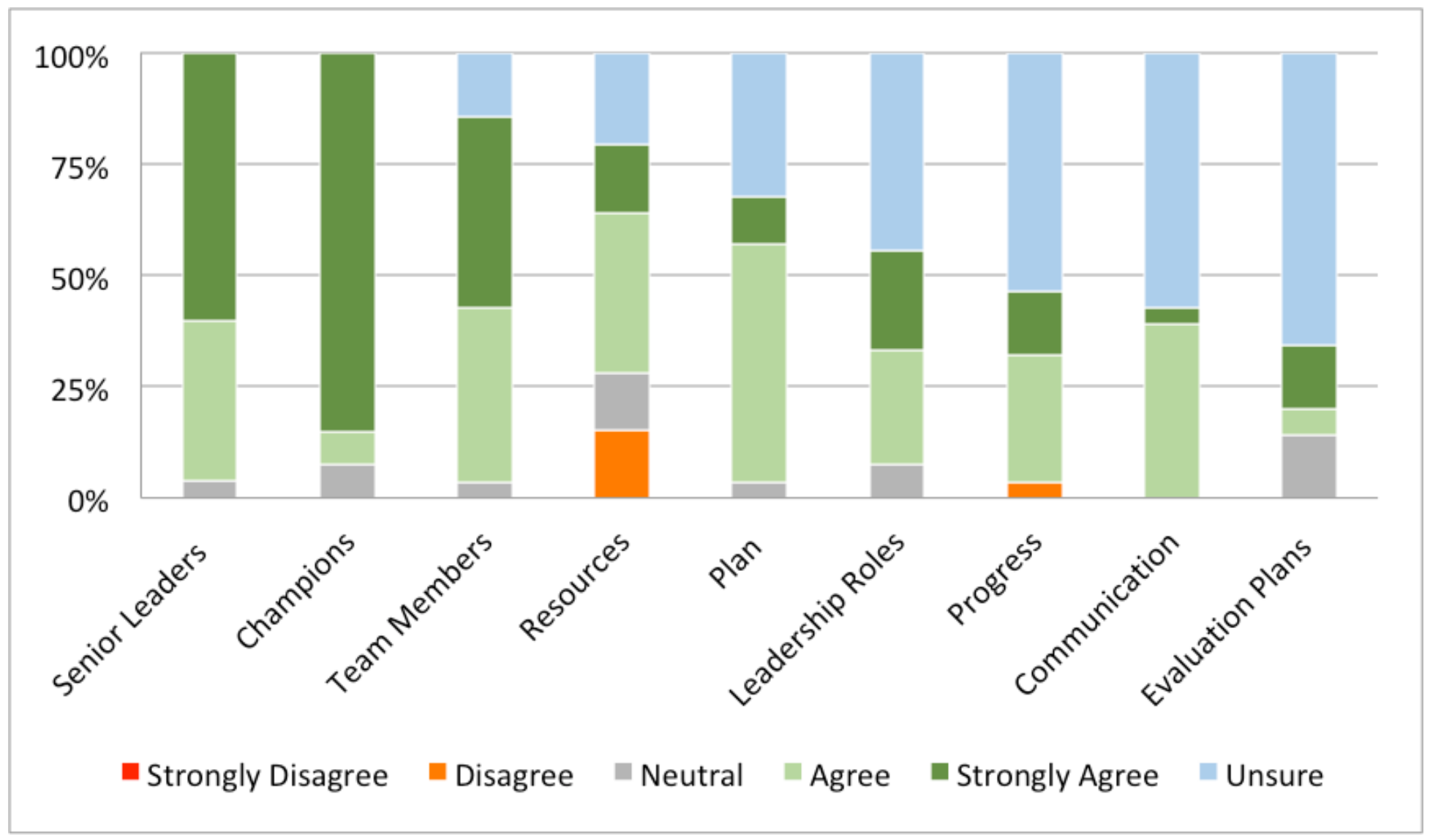

Figure 1

Participant perceptions of the way in which evidence was facilitated for Specialized Vestibular Physiotherapy
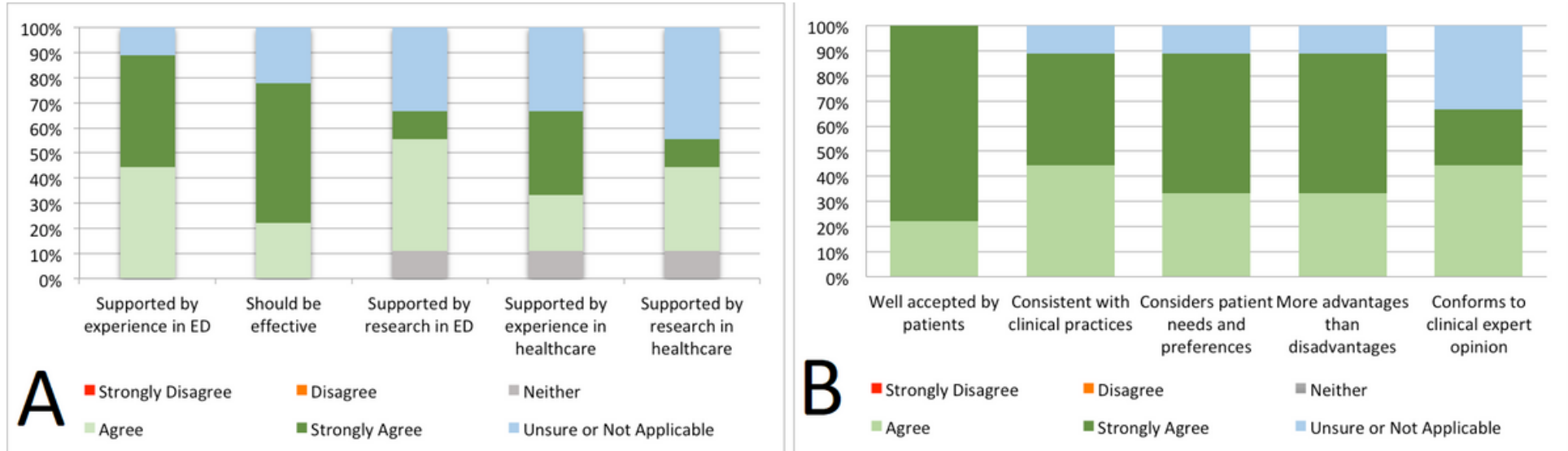

Figure 2

Participant Perceptions of Specialized Vestibular Physiotherapy 


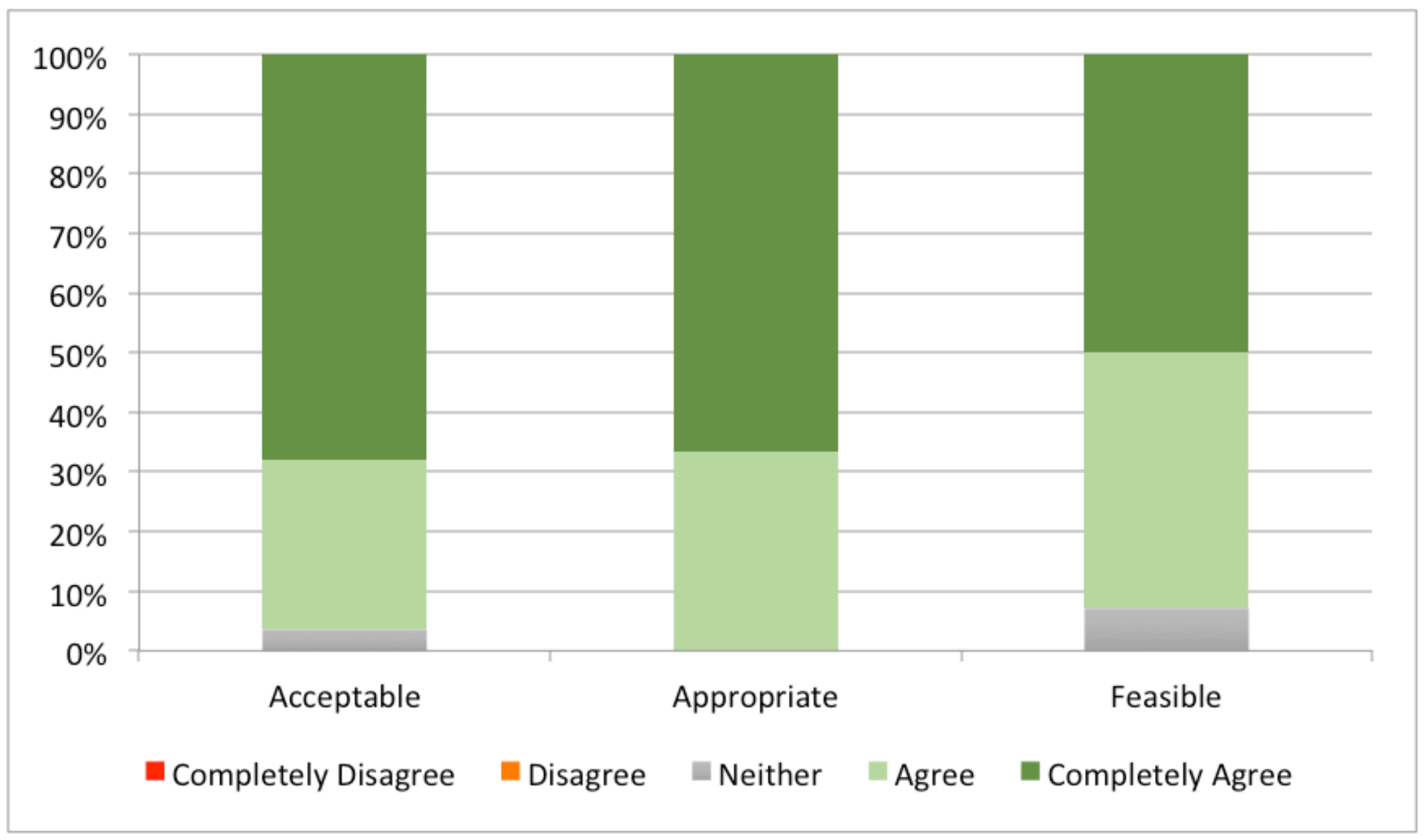

Figure 3

Recipient Perceptions of the Acceptable, Appropriateness and Feasibility of Specialized Vestibular Physiotherapy

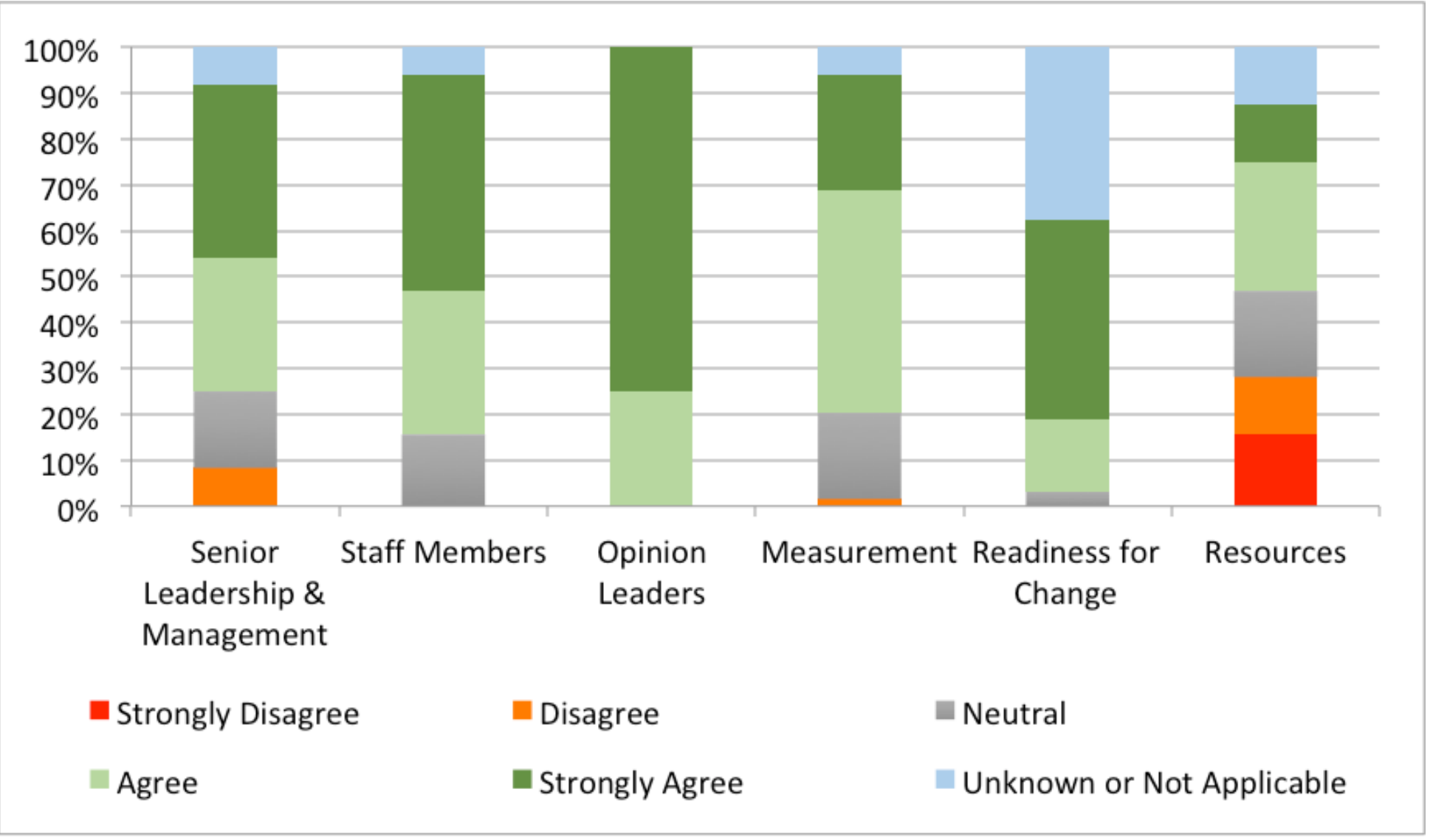


Figure 4

Participant Perceptions of the Context for Specialized Vestibular Physiotherapy

\section{Supplementary Files}

This is a list of supplementary files associated with this preprint. Click to download.

- ImplementationScienceCommunicationAdditionalFile1.docx

- ImplementationScienceCommunicationAdditionalfile2.docx

- ImplementationScienceCommunicationsAdditionalfile3.docx

- ImplementationScienceCommunicationsAdditionalfile4.docx

- ImplementationScienceAdditionalFile5.docx 